\title{
AN INEQUALITY ON DOUBLY STOCHASTIC MATRICES
}

\section{J. EVERETT}

1. Introduction. A problem of van der Waerden concerns the inequality $\operatorname{Per}(X) \geqq n ! / n^{n}$ for a doubly stochastic matrix $X$ of order $n$, i.e., having all elements $x_{i j} \geqq 0$ and all row and column sums unity. The permanent $\operatorname{Per}(X)$ is defined, as a function of the $x_{i j}$, just as is the determinant, except that all signs are taken positive. (Cf. [2].)

We prove it here for the very special case of such a matrix $X$ with one arbitrary row $x_{1}, \cdots, x_{n}$ of non-negative numbers with sum 1 , all $n-1$ remaining rows being $\left(1-x_{1}\right) /(n-1), \cdots,\left(1-x_{n}\right) /(n-1)$. For such an $X$, the above inequality is tantamount to

$$
\sum x_{j}\left\{\left(1-x_{1}\right) \cdots\left(1-x_{n}\right)\right\}_{j} \geqq((n-1) / n)^{n-1},
$$

where the bracket omits the factor $\left(1-x_{j}\right)$.

Even this special case has some interesting consequences. For example, the left side is $-F^{\prime}(1)$, where

$F(t) \equiv\left(1-x_{1} t\right) \cdots\left(1-x_{n} t\right)=1-\sigma_{1} t+\sigma_{2} t^{2}-\cdots+(-1)^{n} \sigma_{n} t^{n}$, so that (1) is equivalent to

$$
\sigma_{1}-2 \sigma_{2}+3 \sigma_{3}-\cdots+(-1)^{n-1} n \sigma_{n} \geqq((n-1) / n)^{n-1},
$$

where the $\sigma_{i}$ are the elementary symmetric functions of non-negative $x_{1}, \cdots, x_{n}$, with $\sigma_{1}=1$.

If any $x_{j}=1$, all others are zero and (1) is trivial. Hence an essentially identical form is

$$
\prod_{1}^{n}\left(1-x_{j}\right) \sum_{1}^{n} x_{j} /\left(1-x_{j}\right) \geqq((n-1) / n)^{n-1},
$$

for $0 \leqq x_{j}<1$, with $\sum_{1}^{n} x_{j}=1$. Consequently, if $\sum_{1}^{\infty} x_{j}=1$, where $0 \leqq x_{j}<1$ for all $j$, then the $n$ numbers $x_{1}, \cdots, x_{n-1}$, and $X_{n}=\sum_{n}^{\infty} x_{f}$ satisfy (3) for $n$ sufficiently large. Since the infinite product and sum converge, we have in the limit

$$
\prod_{1}^{\infty}\left(1-x_{j}\right) \sum_{1}^{\infty} x_{j} /\left(1-x_{j}\right) \geqq e^{-1}
$$

(cf. $[1$, p. 224]). 1963.

Presented to the Society, October 18, 1963; received by the editors October 18, 
2. Proof of an inequality. With little additional trouble we prove a result somewhat stronger than (1), namely the

THEOREM. If for $n \geqq 2, x_{1}, \cdots, x_{n}$ are non-negative real numbers $\leqq 1$ with sum $x \leqq 3 / 2$, then

(5) $S \equiv \sum_{1}^{n} x_{j}\left\{\left(1-x_{1}\right) \cdots\left(1-x_{n}\right)\right\}_{j} \geqq x(1-(x / n))^{n-1} \equiv S_{0}$.

Proof is by induction on $n \geqq 2$. Since $x_{1}\left(1-x_{2}\right)+x_{2}\left(1-x_{1}\right)=x-2 x_{1} x_{2}$ $\geqq x-2(x / 2)^{2}$ for arbitrary non-negative $x_{j}$ with sum $x$, we fix $n \geqq 3$, and $n$ numbers $x_{j}$ with sum $x \leqq 3 / 2$, so ordered that $1 \geqq x_{1} \geqq \cdots \geqq x_{n}$ $\geqq 0$. Clearly $x_{n} \leqq x / n$. Write

$$
\begin{aligned}
S= & \left(1-x_{n}\right) \sum_{1}^{n-1} x_{j}\left\{\left(1-x_{1}\right) \cdots\left(1-x_{n-1}\right)\right\}_{j} \\
& +x_{n}\left(1-x_{1}\right) \cdots\left(1-x_{n-1}\right) .
\end{aligned}
$$

Define $y_{j}$ by the equation $1-x_{j}=k\left(1-y_{j}\right)$ for $j=1, \cdots, n-1$. where $k=\left(n-1-x+x_{n}\right) /(n-1-x)$ is so chosen that the $y_{j}$ also have sum $x$. Since $n-1-x>0$, we have $k \geqq 1$ and each $y_{j}=\left(1-k^{-1}\right)$ $+k^{-1} x_{j}$ is interpolated between $x_{j}$ and 1 . Thus, by the induction hypothesis,

$$
S^{\prime} \equiv \sum_{1}^{n-1} y_{j}\left\{\left(1-y_{1}\right) \cdots\left(1-y_{n-1}\right)\right\}_{j} \geqq x(1-(x / n-1))^{n-2} \equiv S_{0}^{\prime}
$$

while, by the geometric-arithmetic-mean inequality,

$$
P \equiv\left(1-y_{1}\right) \cdots\left(1-y_{n-1}\right) \leqq((n-1-x) /(n-1))^{n-1} .
$$

Since we may write $x_{j}=y_{j}-(k-1) \cdot\left(1-y_{j}\right)$, we see that

$$
\begin{aligned}
S & =k^{n-2}\left\{\left(1-x_{n}\right)\left[S^{\prime}-(n-1)(k-1) P\right]+k x_{n} P\right\} \\
& =k^{n-2}\left\{\left(1-x_{n}\right) S^{\prime}-P x_{n}\left(x-n x_{n}\right) /(n-1-x)\right\} .
\end{aligned}
$$

Using the bounds on $S^{\prime}$ and $P$,

$S \geqq k^{n-2}\left\{(n-1) x\left(1-x_{n}\right)-x_{n}\left(x-n x_{n}\right)\right\}$

$$
\cdot(n-1-x)^{n-2} /(n-1)^{n-1}
$$

$=\left(n-1-x+x_{n}\right)^{n-2}\left\{(n-1) x\left(1-x_{n}\right)-x_{n}\left(x-n x_{n}\right)\right\} /(n-1)^{n-1}$

$\equiv f\left(x_{n}\right)$.

Since $f(x / n)=S_{0}$, it suffices to show that $f\left(x_{n}\right) \geqq f(x / n)$ on $0 \leqq x_{n}$ $\leqq x / n$. This is apparent graphically from the nature of 


$$
\begin{aligned}
& (n-1)^{n-1} f^{\prime}\left(x_{n}\right) \\
& \quad=n^{2}\left(n-1-x+x_{n}\right)^{n-3}\left\{x_{n}-[x-2(n-1) / n]\right\}\left\{x_{n}-[x / n]\right\},
\end{aligned}
$$

where $n-1-x+x_{n}>0$, and the first of the zeros in square brackets is less than the second. If the first zero is nonpositive, the conclusion is clear. The alternative case can only occur when $n=3$ (i.e., in the first induction step), and it then suffices to know that $f(0)=S_{0}^{\prime} \geqq f(x / n)$ $=S_{0}$ in case $n=3$, which is obvious since $x \leqq 3 / 2$. Q.E.D.

Actually, the inequality $S_{0}^{\prime} \geqq S_{0}$ for all $n \geqq 3,0 \leqq x \leqq 3 / 2$, is a corollary of the theorem, since $x_{1}=\cdots=x_{n-1}=x /(n-1), x_{n}=0$ is an admissible set of values, for which $S=S_{0}^{\prime}$ (cf. (6)).

The upper bound $3 / 2$ on $x$ is the best possible constant value since (5) is false for $n=3, x_{1}=x_{2}=x / 2, x_{3}=0$, with any $x$ on $3 / 2<x \leqq 2$. And of course a sum $x \geqq 2$ allows two $x_{j}$ to be unity and (5) is manifestly false for $n>x$.

One can see from the induction that equality holds in (5) if and only if all $x_{j}=x / n$, except in the one additional case $n=3, x=3 / 2$, with one $x_{j}=0$ and the other two equal to $3 / 4$.

As another consequence of the theorem, we note the

COROLlARY. If for $m \geqq 1, x_{1}, \cdots, x_{m}$ are non-negative with sum $x^{\prime} \leqq 1 / 2$, then

$$
\left(1-x_{1}\right) \cdots\left(1-x_{m}\right)>\left(1+x^{\prime}\right)\left(1-\left(1+x^{\prime}\right) /(m+1)\right)^{m} .
$$

This is clear from (5) and (6) if we set $x_{n}=1$ and take $m=n-1$, $x^{\prime}=x-1$. The application to infinite products is the inequality

$$
\prod_{1}^{\infty}\left(1-x_{j}\right) \geqq\left(1+x^{\prime}\right) / \exp \left(1+x^{\prime}\right)
$$

for $0 \leqq x_{j}$ with $\sum_{1}^{\infty} x_{j}=x^{\prime} \leqq 1 / 2$.

The significance of the theorem for permanents is of course that

$$
\operatorname{Per}(X) \geqq\left(n ! / n^{n}\right)\left[x((n-x) /(n-1))^{n-1}\right],
$$

where $X$ is a matrix with rows as prescribed in $\S 1$, the $x_{j}$ satisfying the conditions of the theorem. Note that this $X$ has column sums 1 , and the factor in square brackets above is the product of its row sums.

3. Remark on the general case. Let $X$ be an arbitrary doubly stochastic matrix and denote by $X_{i}$ the matrix of type in $\$ 1$, based on the $i$ th row of $X$. Then we have the inequalities

$$
(1 / n) \sum \operatorname{Per}\left(X_{i}\right) \geqq\left(\prod \operatorname{Per}\left(X_{i}\right)\right)^{1 / n} \geqq \min \operatorname{Per}\left(X_{i}\right) \geqq n ! / n^{n},
$$


and the desired result would follow if any one of these were less than or equal to $\operatorname{Per}(X)$. Actually one can show, for $n=3$, that

$$
3 \operatorname{Per}(X) \geqq \operatorname{Per}\left(X_{1}\right)+\operatorname{Per}\left(X_{2}\right)+\operatorname{Per}\left(X_{3}\right),
$$

but the method offers no hope of generalization.

Even the analogue of the theorem for two arbitrary rows has interesting applications to infinite products and series which seem to be true in the cases tried, but no proof is in sight.

\section{REFERENCES}

1. K. Knopp, Theory and applications of infinite series, 2nd English ed., Hafner, New York, 1947.

2. H. J. Ryser, Combinatorial mathematics, Carus Math. Monograph \#14, Wiley, New York, 1963.

Los Alamos Scientific Laboratory, University of California

\section{PERFECT CLOSURES OF RINGS AND SCHEMES'}

\section{MARVIN J. GREENBERG}

0 . In [3], Serre has defined the notion of a perfect variety over a field of characteristic $p>0$. Of course, a perfect variety is, in general, not a variety. The appropriate setting is that of schemes [2]. We show how to construct the perfect closure of a scheme, in particular, of a ring $A$, of characteristic $p$. This amounts to showing that the functor $B \rightarrow \operatorname{Hom}(A, B)$ is representable in the category of perfect rings. We do this by the technique of inductive limits.

1. Let $A$ be a ring (meaning commutative associative unitary ring) of characteristic $p>0, p$ a prime; $p$ is thus the smallest positive integer $n$ such that $n a=0$ for all $a \in A$. Then $A$ has a canonical ring endomorphism, denoted $F$, given by

$$
F(a)=a^{p}, \quad a \in A .
$$

Clearly, $F$ commutes with all homomorphisms of rings of characteristic $p$.

We say $A$ is perfect if $F$ is an automorphism of $A$ (so that every element of $A$ has a unique $p$ th root in $A$ ). For example, finite fields

Presented to the Society, January 23, 1964, under the title Perfect closures of a scheme; received by the editors October 22, 1963.

1 Supported by AFOSR-121-63. 